\title{
AN INFINITE INTEGRAL INVOLVING A PRODUCT OF TWO MODIFIED BESSEL FUNCTIONS OF THE SECOND KIND
}

\author{
by T. M. MACROBERT
}

(Received 12th August, 1953)

The formula to be established is

$$
\begin{aligned}
& \int_{0}^{\infty} x^{l-1} K_{m}(x) K_{n}(b / x) d x \\
& =\sum_{n,-n} 2^{l-2 n-3} \Gamma(-n) \Gamma\left(\frac{l+m-n}{2}\right) \Gamma\left(\frac{l-m-n}{2}\right) b^{n} F\left(; 1+n, 1-\frac{l+m-n}{2}, 1-\frac{l-m-n}{2} ; \frac{b^{2}}{16}\right) \\
& \quad+\sum_{m,-m} 2^{-l-2 m-3} \Gamma(-m) \Gamma\left(\frac{-l-m+n}{2}\right) \Gamma\left(\frac{-l-m-n}{2}\right) b^{l+m} \\
& \quad \times F\left(; 1+m, 1+\frac{l+m-n}{2}, 1+\frac{l+m+n}{2} ; \frac{b^{2}}{16}\right), \ldots \ldots \ldots \ldots \ldots \ldots \ldots \ldots \ldots \ldots \ldots(1)
\end{aligned}
$$

where $l, m, n$ are any numbers real or complex and $R(b)>0$. A similar result, involving Bessel Functions of the First Kind, was obtained by Hanumanta Rao [Mess. of Maths., XLVII. (1918), pp. 134-137].

The proof is on the same lines as that of Rao. The function $K_{n}(x)$ satisfies the equation

$$
x^{2} y^{\prime \prime}+x y^{\prime}-\left(n^{2}+x^{2}\right) y=0 .
$$

It should also be noted that, if $n$ is not integral

$$
K_{n}(x)=\frac{\pi}{2 \sin n \pi}\left\{I_{-n}(x)-I_{n}(x)\right\}
$$

and that, if $R(l \pm m)>0$,

$$
\int_{0}^{\infty} x^{l-1} K_{m}(x) d x=2^{l-2} \Gamma\left(\frac{l+m}{2}\right) \Gamma\left(\frac{l-m}{2}\right)
$$

Denote the integral by $I$; then

$$
\begin{aligned}
\frac{d I}{d b}=\int_{0}^{\infty} x^{l-2} K_{m}(x) K_{n}^{\prime}(b / x) d x & =-\frac{1}{b}\left[x^{l} K_{m}(x) K_{n}(b / x)\right]_{0}^{\infty} \\
& +\frac{l}{b} \int_{0}^{\infty} x^{l-1} K_{m}(x) K_{n}(b / x) d x+\frac{1}{b} \int_{0}^{\infty} x^{l} K_{m}^{\prime}(x) K_{n}(b / x) d x
\end{aligned}
$$

Therefore

$$
b \frac{d I}{d b}-l I=\int_{0}^{\infty} x^{l} K_{m}^{\prime}(x) K_{n}(b / x) d x
$$

and

$$
\begin{gathered}
b \frac{d^{2} I}{d b^{2}}+(1-l) \frac{d I}{d b}=\int_{0}^{\infty} x^{l-1} K_{m}^{\prime}(x) K_{n}^{\prime}(b / x) d x \\
=-\frac{1}{b}\left[x^{l+1} K_{m}^{\prime}(x) K_{n}(b / x)\right]_{0}^{\infty}+\frac{l+1}{b} \int_{0}^{\infty} x^{l} K_{m}^{\prime}(x) K_{n}(b / x) d x+\frac{1}{b} \int_{0}^{\infty} x^{l+1} K_{m}^{\prime \prime}(x) K_{n}(b / x) d x \\
=\frac{l+1}{b}\left\{b \frac{d I}{d b}-l I\right\}+\frac{1}{b} \int_{0}^{\infty} x^{l-1}\left\{\left(x^{2}+m^{2}\right) K_{m}(x)-x K_{m}^{\prime}(x)\right\} K_{n}(b / x) d x,
\end{gathered}
$$


by (2). Hence

$$
b^{2} \frac{d^{2} I}{d b^{2}}+(1-2 l) b \frac{d I}{d b}+\left(l^{2}-m^{2}\right) I=J,
$$

where $J=\int_{0}^{\infty} x^{l+1} K_{m}(x) K_{n}(b / x) d x$.

Again

$$
\frac{d J}{d b}=\int_{0}^{\infty} x^{l} K_{m}(x) K_{n}^{\prime}(b / x) d x
$$

and

$$
\begin{aligned}
\frac{d^{2} J}{d b^{2}} & =\int_{0}^{\infty} x^{l-1} K_{m}(x) K_{n}^{\prime \prime}(b / x) d x \\
& =\frac{1}{b^{2}} \int_{0}^{\infty} x^{b+1} K_{m}(x)\left\{\left(\frac{b^{2}}{x^{2}}+n^{2}\right) K_{n}\left(\frac{b}{x}\right)-\frac{b}{x} K_{n}^{\prime}\left(\frac{b}{x}\right)\right\} d x \\
& =I+\frac{n^{2}}{b^{2}} J-\frac{1}{b} \frac{d J}{d b}
\end{aligned}
$$

Now, from $(A)$,

$$
\begin{aligned}
& \frac{d J}{d b}=b^{2} \frac{d^{3} I}{d b^{3}}+(3-2 l) b \frac{d^{2} I}{d b^{2}}+\left(1-2 l+l^{2}-m^{2}\right) \frac{d I}{d b} \\
& \frac{d^{2} J}{d b^{2}}=b^{2} \frac{d^{4} I}{d b^{4}}+(5-2 l) b \frac{d^{3} I}{d b^{3}}+\left(4-4 l+l^{2}-m^{2}\right) \frac{d^{2} I}{d b^{2}} .
\end{aligned}
$$

and

Hence, after some simplification,

$$
\begin{aligned}
b^{4} \frac{d^{4} I}{d b^{4}} & +(6-2 l) b^{3} \frac{d^{3} I}{d b^{3}}+\left(7-6 l+l^{2}-m^{2}-n^{2}\right) b^{2} \frac{d^{2} I}{d b^{2}} \\
& +\left(1-2 l+2 l n^{2}+l^{2}-m^{2}-n^{2}\right) b \frac{d I}{d b}+\left(m^{2}-l^{2}\right) n^{2} I=b^{2} I
\end{aligned}
$$

Next let $I=\sum_{\nu=0}^{\infty} c_{\nu} b^{\rho+\nu}$; then, on substituting in (B), the coefficient of $c_{0} b^{\rho}$ is

$$
\begin{aligned}
\rho(\rho-1)( & (\rho-2)(\rho-3)+(6-2 l) \rho(\rho-1)(\rho-2)+\left(7-6 l+l^{2}-m^{2}-n^{2}\right) \rho(\rho-1) \\
& +\left(1-2 l+2 l n^{2}+l^{2}-m^{2}-n^{2}\right) \rho+\left(m^{2}-l^{2}\right) n^{2} \\
= & \rho^{4}-2 l \rho^{3}+\left(l^{2}-m^{2}-n^{2}\right) \rho^{2}+2 l n^{2} \rho+\left(m^{2}-l^{2}\right) n^{2} \\
= & (\rho-n)(\rho+n)(\rho-l-m)(\rho-l+m) .
\end{aligned}
$$

Thus the indicial equation gives

$$
\rho=n,-n, l+m, l-m .
$$

If $c_{1}=0$ then all the $c$ 's with odd suffixes vanish, while, for $\nu=0,1,2, \ldots$,

Hence

$$
c_{2 \nu+2}(\rho+2 \nu+2-n)(\rho+2 \nu+2+n)(\rho+2 \nu+2-l-m)(\rho+2 \nu+2-l+m)=c_{2 v} \text {. }
$$

$$
I=A b^{n} P+B b^{-n} Q+C b^{l+m} R+D b^{l-m} S,
$$

where $P, Q, R, S$ are the generalised hypergeometric functions on the right of (1), taken in order.

In determining the values of the coefficients $A, B, C, D$, it should be noticed that the value of the integral is unaltered if the sign of $m$ or of $n$ is altered. Let it be assumed that $R(n)$ is positive, multiply by $b^{n}$ and let $b \rightarrow 0$; then, if $R\left(l_{ \pm} m+n\right)>0$, from (3),

and therefore, from (4),

$$
\frac{\pi}{2 \sin n \pi} \frac{2^{n}}{\Gamma(1-n)} \int_{0}^{\infty} x^{l+n-1} K_{m}(x) d x=B
$$

$$
B=2^{l+2 n-3} \Gamma(n) \Gamma\left(\frac{l+m+n}{2}\right) \Gamma\left(\frac{l-m+n}{2}\right) .
$$


Again, in $I$ replace $x$ by $b / x$, and it becomes

$$
b^{2} \int_{0}^{\infty} x^{-l-1} K_{m}(b / x) K_{n}(x) d x
$$

From this, assuming that $R(m)$ is positive, on multiplying by $b^{m-l}$ and making $b \rightarrow 0$, it is found that, if $R(-l+m \pm n)>0$,

$$
D=2^{-l+2 m-3} \Gamma(m) \Gamma\left(\frac{-l+m+n}{2}\right) \Gamma\left(\frac{-l+m-n}{2}\right) .
$$

Since $I, R$ and $S$ are symmetrical in $n$ and $-n$, and since $b^{n} P$ becomes $b^{-n} Q$ when $n$ and $-n$ are interchanged, it follows that, if $R(l \pm m+n)>0, A$ is equal to $B$ with $n$ and $-n$ interchanged, and that $C$ and $D$ are symmetrical in $n$ and $-n$.

Similarly, when $R(-l+m \pm n)>0, C$ is $D$ with $m$ and $-m$ interchanged.

Now, from the continuity of the functions with respect to $l$, if $R(m)$ and $R(n)$ are taken to be positive, all these values of $A, B, C$, and $D$ are valid when $R(l)=R(m-n)$. But all the functions in equation (1) are holomorphic in $l$ for all real or complex values of $l$. Hence, by analytical continuation, formula (1) holds for all values of $l$.

Note. On putting $l=1, m=n$, Hardy's formula

$$
\int_{0}^{\infty} K_{n}(x) K_{n}(b / x) d x=\pi K_{2 n}(2 \sqrt{ } b)
$$

where $R(b)>0$, is obtained.

University of Glasgow 